\title{
ROLE OF THE SUBSTITUTIONAL OXYGEN DONOR IN THE RESIDUAL N-TYPE CONDUCTIVITY IN GaN
}

\author{
W.M. Chen, I.A. Buyanova, ${ }^{*}$ Mt. Wagner, ${ }^{*}$ B. Monemar, ${ }^{*}$ J.L. Lindström, ${ }^{* *}$ H. Amano ${ }^{* * *}$, I. \\ Akasaki $^{* * *}$ \\ *Department of Physics and Measurement Technology, Linköping University, S-581 83 \\ Linköping, SWEDEN,wmc@ifm.liu.se \\ ${ }^{* *}$ Swedish Defense Research Establishment, Box 1165, S-581 11 Linköping, SWEDEN \\ **** Department of Electrical and Electronic Engineering, Meijo University, 1-501 Shiogamaguchi, \\ Tempaku-ku, Nagoya 468, JAPAN
}

Cite this article as: MRS Internet J. Nitride Semicond. Res. 4S1, G5.4(1999)

\begin{abstract}
A detailed photoluminescence (PL) study reveals a striking similarity in local vibrational properties of a defect center in $\mathrm{GaN}$ as compared to that for the substitutional $\mathrm{O}_{\mathrm{P}}$ donor in $\mathrm{GaP}$. This observation could be interpreted as if the center is in fact related to the substitutional oxygen donor in GaN. The deep-level nature experimentally determined for the defect center calls for caution of a commonly referred model that the substitutional oxygen donor is responsible for the residual n-type conductivity in $\mathrm{GaN}$.
\end{abstract}

\section{INTRODUCTION}

Oxygen is one of the most common impurities which is frequently present during growth and processing steps as a contaminant, and can be unintentionally incorporated into many semiconductors including III-nitrides. This uncontrolled introduction of the oxygen impurity may affect and sometimes degrade the performance of semiconductor devices. The understanding of the role of oxygen in altering the material properties, and eventually a control of its introduction, are therefore of crucial importance for a reliable device operation. The substitutional oxygen donor has recently been suggested to be responsible for a long-standing materials problem - the residual n-type conductivity in oxygen-containing GaN. This conclusion was based both on theoretical calculations of the formation energy and the electronic structure of the oxygen donor in $\mathrm{GaN}[1,2]$ and on experimental observation of a strong correlation between the oxygen content in the material and the free electron concentration [3]. Though there is so far no direct experimental evidence available as to the exact form of the oxygen center and its geometric structure, the suggestion seemed to be reasonable and consistent until recently, when a new photoluminescence emission (denoted as the 0.88-eV PL below) of a deep center has provided microscopic information on local vibrations of the defect.

In this paper, we shall demonstrate that these local vibrations resemble those reported earlier for the substitutional $\mathrm{O}_{\mathrm{P}}$ donor in GaP. This striking similarity, together with the electronic structure revealed from a detailed PL study of the deep center, has led us to suggest that the substitutional $\mathrm{O}_{\mathrm{N}}$ donor in $\mathrm{GaN}$ could be a deep donor, and thus to call for caution in assessing its role in the n-type conductivity of the material.

\section{EXPERIMENTAL}


The samples studied in this work were a variety of wurtzite GaN layers, typically 1- 200 $\mu \mathrm{m}$ thick, grown by hydride vapor phase epitaxy (HVPE) or metal organic chemical vapor deposition (MOCVD) on (0001) sapphire or 6H SiC substrates. The conductivity of the samples before electron irradiation varies over a wide range from highly $\mathrm{n}$-type $\left([\mathrm{n}] \sim\right.$ higher $\left.10^{18} \mathrm{~cm}^{-3}\right)$, highly compensated $\left([\mathrm{n}] \sim 10^{16}\right.$ - lower $\left.10^{17} \mathrm{~cm}^{-3}\right)$ to highly p-type $\left([\mathrm{p}] \sim\right.$ higher $\left.10^{17} \mathrm{~cm}^{-3}\right)$. These samples were irradiated by $2.5-\mathrm{MeV}$ electrons at room temperature with a dose of $1 \times 10^{17}$ $4 \times 10^{18} \mathrm{~cm}^{-2}$, to enhance the $0.88-\mathrm{eV}$ PL intensity.

In PL experiments, the samples were excited by the 334 or $351 \mathrm{~nm}$ UV lines of an Argon ion laser. The resulting PL was spectrally dispersed by a $0.85-\mathrm{m}$ double grating monochromator, and monitored by a cooled Ge detector. In temperature dependent PL studies, the sample temperature was varied between $1.5 \mathrm{~K}$ and room temperature. A magnetic field up to $14 \mathrm{~T}$ was applied in Zeeman studies of the PL spectra, with the aid of a superconducting magnet.

\section{RESULTS AND DISCUSSION}

\section{$\underline{\text { Defect Models }}$}

A typical low-temperature $0.88-\mathrm{eV}$ PL spectrum in $\mathrm{GaN}$ is shown as the upper curve in Fig.1. The observation of this PL emission in electron irradiated GaN was first reported by Linde et al [4]. It can be observed in all the GaN epilayers studied, indicating that it is related to either a native defect or a common contaminant. This $0.88-\mathrm{eV}$ PL is characterized by a relatively sharp no phonon (NP) line near $0.88 \mathrm{eV}$, accompanied by a rich structure of phonon replicas on the lower energy side. A detailed inspection of the phonon replicas reveals many local phonon modes, apart from the known lattice phonons. This in fact represents a rare case in $\mathrm{GaN}$ when so many local phonons have been well resolved. What is more unusual and

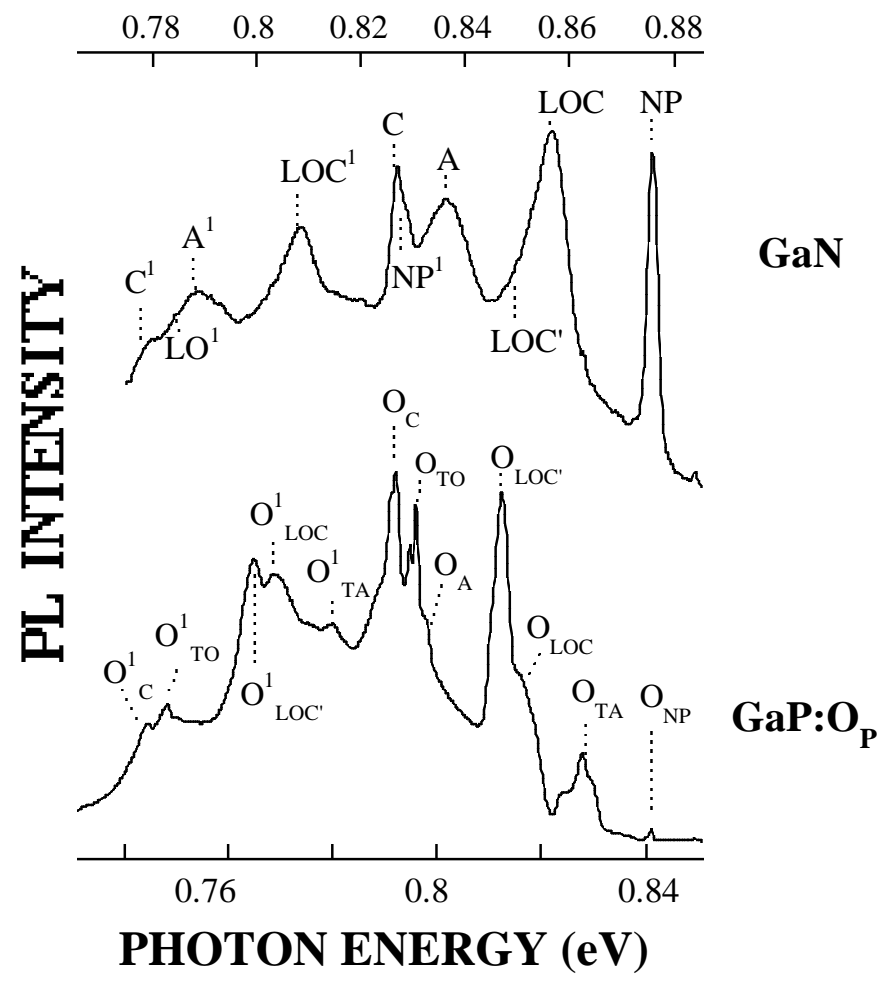

Fig. 1 The $0.88-\mathrm{eV}$ PL in GaN (the upper curve) and the 0.841 $\mathrm{eV}$ in $\mathrm{GaP}$ (the lower curve) taken at $1.6 \mathrm{~K}$. The horizontal scales for the photon energy are shifted relatively to line up the NP lines of the two spectra, for easy comparison. The definitions of the notations follow mostly those commonly used in the literature [5]. NP for no-phonon, TA for transverse acoustic phonon of the host lattice, TO for transverse optical phonon of the host lattice, LO for longitudinal optical phonon of the host lattice, LOC, LOC', A and C for local phonons. Superscript 1 denotes the involvement of an additional phonon $\hbar w_{0}$. 
Table I. A summary of the local phonon energies from the 0.841-eV PL in GaP and the 0.88-eV PL in $\mathrm{GaN}$. The definitions of the notations for local phonons, i.e. LOC, LOC', A and C, follow mostly those commonly used in the literature [5]. Superscript 1 denotes the involvement of an additional phonon $\hbar w_{0}$.

\begin{tabular}{|c|c|c|}
\hline \multirow{2}{*}{$\begin{array}{c}\text { Local } \\
\text { Phonon Modes }\end{array}$} & \multicolumn{2}{|c|}{ Energy Shifts (meV) } \\
\hline & $\begin{array}{c}\text { 0.841-eV PL } \\
\text { in GaP }\end{array}$ & $\begin{array}{c}\text { 0.88-eV PL } \\
\text { in GaN }\end{array}$ \\
\hline $\mathrm{LOC}$ & 24.7 & 19.1 \\
\hline LOC' $^{\prime}$ & 28.4 & 26.2 \\
\hline $\mathrm{A}$ & 43.0 & 39.6 \\
\hline $\mathrm{C}$ & 48.7 & 49.1 \\
\hline $\mathrm{LOC}^{1}$ & $24.7+47.5$ & $19.1+48$ \\
\hline LOC $^{\prime 1}$ & $28.4+47.5$ & $26.2+48$ \\
\hline $\mathrm{A}^{1}$ & $43.0+47.5$ & $39.6+48$ \\
\hline $\mathrm{C}^{1}$ & $48.7+47.5$ & $49.1+48$ \\
\hline
\end{tabular}

surprising is that a striking similarity has been found between these local phonons in $\mathrm{GaN}$ and those observed in the $\mathrm{O}_{\mathrm{P}}$ related $0.841-\mathrm{eV}$ PL emission in GaP. To illustrate that, the $0.841-\mathrm{eV}$ $\mathrm{PL}$ in $\mathrm{GaP}$ is also shown in Fig. 1 (the lower curve), but shifted in energy to line up the NP lines of the two PL emissions. The local phonon modes involved in the two cases are listed in Table I for an easy comparison. It is rather obvious that nearly all the local phonons are indeed similar in energy for these two semiconductors, excluding the lattice phonons which are different between them. This fact can either be interpreted as a pure coincidence or it can indicate that a similar defect is involved. Since this seems to be the only case, to our knowledge, that such a striking similarity in nearly all local phonon modes was found between two defect systems in semiconductors, a pure coincidence seems to be rather unlikely. If this is the case, the knowledge on physical properties of the $0.841-\mathrm{eV}$ PL center in GaP can be inferred to the 0.88eV PL center in GaN.

Fortunately, the 0.841-eV PL in GaP was the subject of intensive investigations during the seventies and eighties, and a great deal of its properties have been established. The origin of the 0.841-eV PL has unambiguously been associated with the radiative transition between the valleyorbit states $1 \mathrm{~s}(\mathrm{E}) \rightarrow 1 \mathrm{~s}\left(\mathrm{~A}_{1}\right)$ of the isolated, substitutional $\mathrm{O}_{\mathrm{P}}$ donor in its neutral charge state [5], as the final step of the electron capture to the positively charged $\mathrm{O}_{\mathrm{P}}$ donor. The phonon sideband on the low energy side of the 0.841-eV PL (Fig.1 and Table I) has in fact been regarded as characteristic for the $\mathrm{O}_{\mathrm{P}}$ donor in GaP. From the observed isotope shifts $\left(\mathrm{O}^{16} \rightarrow \mathrm{O}^{18}\right)$ in the energy of $\mathrm{O}_{\mathrm{LOC}}$ and $\mathrm{O}_{\mathrm{LOC}}$, for example, they are believed to be primarily dominated by the motion of the $\mathrm{O}$ atom [5]. To possess similar local vibrations in $\mathrm{GaN}$, the corresponding defect should have similar local surroundings and bonding as the $\mathrm{O}_{\mathrm{P}}$ donor in $\mathrm{GaP}$, i.e. one oxygen and four gallium atoms, at least within the nearest neighbor shell. The only candidate is then the substitutional $\mathrm{O}_{N}$ donor in $\mathrm{GaN}$, either an isolated donor or a complex involving $\mathrm{O}_{\mathrm{N}}$, as the defect responsible for the 0.88-eV PL. Due to the striking similarity between the local phonons observed in the 0.88-eV PL in GaN and the 0.841-eV PL in GaP, the other constituent(s) of the complex defect should reside beyond the nearest neighbor shell.

It is interesting to note that the energies of many other localized phonons (such as $\mathrm{O}_{\mathrm{A}}, \mathrm{O}_{\mathrm{C}}$ and $h w_{0}$ ), not fully understood so far for the $\mathrm{O}_{\mathrm{P}}$ donor in $\mathrm{GaP}$, are also remarkably similar between the $\mathrm{O}_{\mathrm{P}}$ donor in $\mathrm{GaP}$ and the $\mathrm{O}_{\mathrm{N}}$ donor in $\mathrm{GaN}$ (Table $\mathrm{I}$ ).

The role of post-growth electron irradiation in enhancing the $0.88-\mathrm{eV}$ PL intensity in GaN is not yet fully understood and requires further studies. A change in the Fermi level position by the electron irradiation might be one of the explanations, which has been shown to be important for 
the observation of the $0.841-\mathrm{eV}$ PL emission in the case of the $\mathrm{O}_{\mathrm{P}}$ donor in GaP. Other possibilities include an irradiation induced formation or activation of the corresponding PL center.

\section{$\underline{\text { Recombination Models }}$}

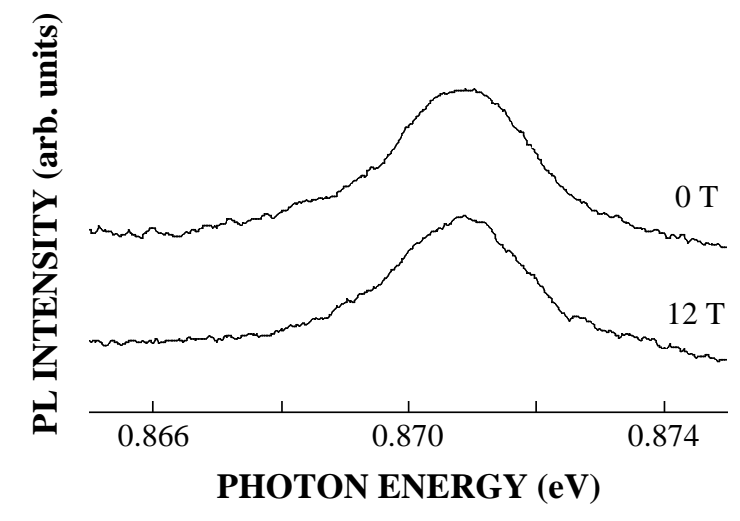

Fig.2 The 0.88-eV PL spectra in GaN taken at $2 \mathrm{~K}$ in the vicinity of the NP line, at magnetic fields of 0 and $12 \mathrm{~T}$.

The recombination mechanism of the $0.88-\mathrm{eV}$ PL in GaN could thus be explained in terms of an electronic transition related to the substitutional $\mathrm{O}_{\mathrm{N}}$ center, either an isolated donor or a complex involving $\mathrm{O}_{\mathrm{N}}$.

Within the first model of an isolated, substitutional $\mathrm{O}_{\mathrm{N}}$ donor, two possible mechanisms responsible for the $0.88-\mathrm{eV}$ PL can be a transition between an excited state and the ground state of the $\mathrm{O}_{\mathrm{N}}$ donor in its neutral charged state (in the same fashion as the $0.841-\mathrm{eV}$ PL in GaP), or in its negatively charged state. In the latter case, the capture of the second electron to the $\mathrm{O}_{\mathrm{N}}$ donor is assumed not to cause a large lattice relaxation of the defect due to a severe softening of the Ga-O bonds so that the local phonon modes are reasonably well preserved. This is not the case for the $\mathrm{O}_{\mathrm{P}}$ donor in $\mathrm{GaP}$, of which a large lattice relaxation was observed upon the capture of the second electron [5]. The situation may be quite different in $\mathrm{GaN}$ in the presence of a lower-symmetry wurtzite crystal field and a more rigid and compact crystal lattice.

To determine whether the $0.88-\mathrm{eV}$ PL originates from an electronic transition within the neutral or negatively charged state of the isolated $\mathrm{O}_{\mathrm{N}}$ donor, a detailed magnetooptical (Zeeman) study was carried out. This was based on the expectations that a different behavior between the two charge states should be observed upon application of an external magnetic field. In the neutral charge state, only one electron is bound to the $\mathrm{O}_{N}$ donor. Both the 1 s ground state and the excited states are at least spin degenerate and are expected to split in the magnetic field. In the case of the negatively charged state two electrons are bound to the donor. The ground state is then expected to be a non-degenerate singlet, where the spins of the two electrons are paired off. The excited states can, however, be either degenerate or non-degenerate. In Fig.2, PL spectra in a close vicinity of the NP line are shown with or without applying a magnetic field. As it is clear from the figure, no noticeable splitting can be detected when the magnetic field was along the caxis or was tilted by an angle with respect to the c-axis. It should be noted that the size of the expected Zeeman splitting, assuming a common value of $\mathrm{g}$ close to $2 \mathrm{in} \mathrm{GaN}$, is comparable to the PL linewidth and is expected to be observed in the experiments. Therefore the absence of any splitting or broadening can be taken as a piece of strong evidence that the recombination occurs between the singlets. In other words, both the excited and ground state are non-degenerate. This experimental fact excludes the possibility of the neutral charge state of the $\mathrm{O}_{\mathrm{N}}$ donor as responsible for the $0.88-\mathrm{eV}$ PL, leaving the negatively charged state as a possible model.

In the second model that an internal electronic transition at a complex defect involving the $\mathrm{O}_{\mathrm{N}}$ donor is responsible for the $0.88-\mathrm{eV}$ PL, both the ground and excited state should be singlets to agree with the Zeeman results. 


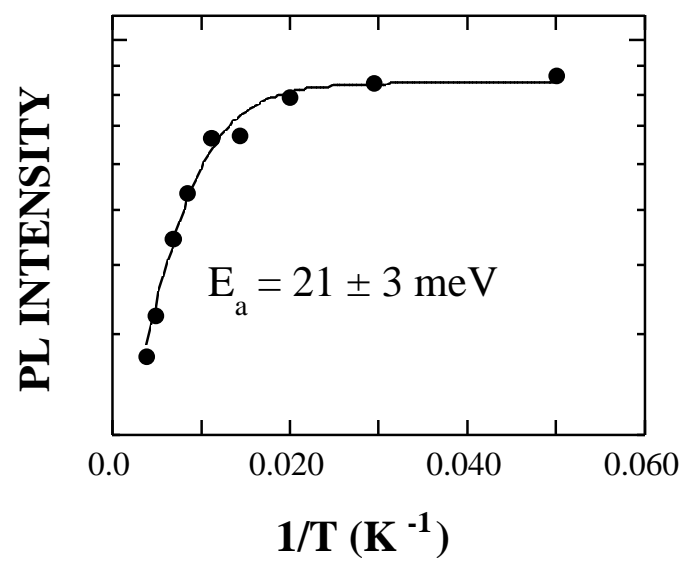

Fig.3 An Arrhenius plot of the integrated intensity of the $0.88-\mathrm{eV}$ PL emission. The activation energy $\mathrm{E}_{\mathrm{a}}$ for the thermal quenching can be estimated.

\section{Binding Energy}

In order to assess the role of the $\mathrm{O}_{\mathrm{N}}$ donor in $\mathrm{GaN}$ in the residual n-type conductivity, the knowledge on the binding energy of the $\mathrm{O}_{\mathrm{N}}$ donor in $\mathrm{GaN}$ is essential. From the PL transition energy, the energy separation between the ground and excited state can be determined to be around $0.88 \mathrm{eV}$. The determination of the binding energy thus requires the knowledge of the ionization energy from the excited state to the bottom of the conduction band. This can be done by temperature dependent studies of the PL intensity, where the thermal quenching of the PL is a direct result of the thermal ionization process. In Fig. 3 such a thermal quenching spectrum of the $0.88-\mathrm{eV} \mathrm{PL}$ is

shown. The activation energy of the thermal quenching, i.e. the energy separation between the excited state to the bottom of the conduction band, was estimated to be about $21 \mathrm{meV}$. It should be pointed out that this value is fairly close to the binding energy expected for the effective-mass donor electron in GaN. Adding the value of $0.88 \mathrm{eV}$ between the ground and excited state, it totals a value of approximately $0.9 \mathrm{eV}$ for the binding energy of the electron for the $0.88-\mathrm{eV} \mathrm{PL}$ center.

In the model of the isolated, negatively charged $\mathrm{O}_{\mathrm{N}}$ donor, this corresponds to the binding energy of the second electron at the donor. Or equivalently, the (-/0) level of the $\mathrm{O}_{\mathrm{N}}$ donor in $\mathrm{GaN}$ is located at $\sim \mathrm{E}_{\mathrm{c}}-0.9 \mathrm{eV}$. Disregarding the possibility of a negative- $\mathrm{U}$ effect, granted by the assumption of the absence of a large lattice distortion discussed above, the binding energy of the first electron at the $\mathrm{O}_{\mathrm{N}}$ donor in $\mathrm{GaN}$ should be larger than $0.9 \mathrm{eV}$. Within this model, the isolated, substitutional $\mathrm{O}_{\mathrm{N}}$ donor is too deep to be ionized at room temperature to account for the residual n-type conductivity in GaN. Other origins must therefore be sought in this case. From a strong correlation between the oxygen content and the free electron concentration [3], however, it seems reasonable to assume that the shallow donor(s) responsible for the residual n-type conductivity in $\mathrm{O}$-containing $\mathrm{GaN}$ should somehow be related to oxygen. It can be an O-related defect, other than the isolated substitutional $\mathrm{O}_{\mathrm{N}}$ donor, such as an oxygen interstitial or a complex involving substitutional or interstitial oxygen.

Within the second model of a complex involving the $\mathrm{O}_{\mathrm{N}}$ donor, the binding energy of the isolated $\mathrm{O}_{\mathrm{N}}$ donor can not be deduced in a similar way. The value of $0.9 \mathrm{eV}$ determined for the ionization energy from the ground state of the complex can be largely contributed by the potential of the $\mathrm{O}_{\mathrm{N}}$ donor. A quantitative analysis is, however, hardly possible. Based on the experimental observation that the electronic transition is strongly coupled to the local vibrations, a major part of the defect wavefunction should be localized near the $\mathrm{O}_{\mathrm{N}}$ donor. This implies that the $\mathrm{O}_{\mathrm{N}}$ donor is most likely a deep donor, and can thus not be responsible for the residual n-type conductivity in $\mathrm{GaN}$.

\section{SUMMARY}


A striking similarity in the local vibrational properties between the $0.88-\mathrm{eV}$ PL in various wurtzite $\mathrm{GaN}$ samples and the $0.841-\mathrm{eV} \mathrm{PL}$ in $\mathrm{GaP}$ has been revealed from a detailed photoluminescence study. This similarity, together with the earlier unambiguous identification of these local vibrations originating from the isolated, substitutional $\mathrm{O}_{\mathrm{P}}$ donor in $\mathrm{GaP}$, suggests that the $0.88-\mathrm{eV}$ PL in $\mathrm{GaN}$ is due to an electronic transition related to the substitutional $\mathrm{O}_{\mathrm{N}}$ donor. Though the exact geometric structure of the center can not be determined at present, possible models have been discussed in terms of the isolated $\mathrm{O}_{\mathrm{N}}$ donor or a complex defect involving the $\mathrm{O}_{\mathrm{N}}$ donor. Within the model of the isolated $\mathrm{O}_{\mathrm{N}}$ donor, the $0.88-\mathrm{eV}$ PL could originate from the capture of the first or second electron to the $\mathrm{O}_{\mathrm{N}}$ donor. The Zeeman studies of the electronic structure of the defect states involved in the recombination seem to rule out the possibility that the first electron capture could be responsible. In combination with the temperature dependent studies of the $0.88-\mathrm{eV}$ PL, the isolated $\mathrm{O}_{\mathrm{N}}$ donor is concluded to be a deep donor with a large binding energy large than $0.9 \mathrm{eV}$. It is thus too deep to be responsible for the high residual n-type conductivity in GaN. Within the second model (i.e. an $\mathrm{O}_{\mathrm{N}}$-related complex defect giving rise to the $0.88-\mathrm{eV}$ PL), the other constituent(s) of the complex defect should reside beyond the nearest neighbor shell to account for the similarity in the local vibrations between $\mathrm{O}_{N}$ in $\mathrm{GaN}$ and $\mathrm{O}_{\mathrm{P}}$ in $\mathrm{GaP}$. A strong coupling of the electronic transition with the local vibrations also seems to indicate that the $\mathrm{O}_{\mathrm{N}}$ donor in $\mathrm{GaN}$ is a deep donor. This work therefore calls for caution with the commonly referred model, where the $\mathrm{O}_{\mathrm{N}}$ donor is suggested to be responsible for the residual $\mathrm{n}$ type conductivity in O-rich GaN.

\section{REFERENCES}

1. J. Neugebauer, C. G. Van de Walle, "Atomic geometry and electronic structure of native defects in GaN", Phys. Rev. B 50 (11), 8067 (1994).

2. Jörg Neugebauer and Chris G. Van de Walle, "Gallium vacancies and the yellow luminescence in GaN”, Appl. Phys. Lett. 69 (4), 503 (1996).

3. G. Wetzel, T. Suski, J. W. Ager, E. R. Weber, E. E. Haller, S. Fischer, B. K. Meyer, R. J. Molnar, P. Perlin, " Pressure induced deep gap state of oxygen in GaN", Phys. Rev. Lett. 78 (20), 3923 (1997).

4. M. Linde, S. F. Uftring, G. D. Watkins, V. Härle and F. Scholz, "Optical detection of magnetic resonance in electron-irradiated GaN", Phys. Rev. B55, R10177 (1997).

5. For a review, see e.g. P.J. Dean “ Oxygen in GaP” in "Deep Centers in Semiconductors" ed. S.T. Pantelides (Gordon and Breach Science Pub. New York, 1986) p.185; P.J. Dean and C.H. Henry, Phys. Rev. 176, 928 (1968). 